\title{
La muerte de Franco y la Transición española a través de la prensa internacional: la visión periodística del Reino Unido, Francia, Italia y Estados Unidos ${ }^{1}$
}

\author{
Ruth RodRIGUEZ-MARTINEZ \\ ruth.rodriguez@upf.edu \\ Universitat Pompeu Fabra \\ Christopher TULLOCH \\ christopher.tulloch@upf.edu \\ Universitat Pompeu Fabra \\ Jaume Guillamet Lloveras \\ jaume.guillamet@upf.edu \\ Universitat Pompeu Fabra
}

Recibido: 5 de marzo de 2014

Aceptado: 22 de septiembre de 2014

\begin{abstract}
Resumen
El propósito de este artículo es analizar el papel y cobertura desempeñada por la prensa internacional tras la muerte de Franco y la posterior Transición a la democracia en España (1975-1978). El periodo sobre el que versa esta investigación comprende la muerte de Franco, el 20 de noviembre de 1975, hasta la ratificación por referéndum del Constitución democrática, el 6 de diciembre de 1978. Los resultados del análisis permiten observar que, a pesar del temor del estallido de una nueva Guerra Civil o la resistencia al cambio de los grupos más conservadores, los once diarios estudiados apoyaron de forma crítica pero incondicional el proceso democrático.

Palabras clave: España, General Franco, Guerra Civil, Transición democrática, corresponsales extranjeros, Rey Juan Carlos, Adolfo Suárez, Francia, Italia, Reino Unido, Estados Unidos.

\section{The Death of Franco and the Spanish Transition to Democracy: the Role and Coverage of the International Press}

\begin{abstract}
The aim of this article is to analyze the role and coverage of the foreign press surrounding the death of Franco and Spain's consequent transition to democracy (1975-1978), at one of the most crucial moments of political change in Southern Europe within the ongoing context of the Cold War. The period under consideration runs from the death of Franco on the 20th of November 1975 to the ratification by referendum of the democratic Constitution on December 6th, 1978. Faced by the threat of a new civil war due to the resistance to change by those who, after winning the war in 1939, paved the way to the Francoist dictatorship, the international press, while still critical unconditionally supported the democratic process, according to the study of the broad coverage carried out by eleven main newspapers in France, Italy, United Kingdom and the United States of America.

Keywords: Spain, General Franco, Civil War, democratic transition, foreign correspondents, King Juan Carlos I, Adolfo Suárez, France, Italy, United Kingdom, United States of America.

1 Los resultados de esta investigación derivan del proyecto de investigación "Noticias internacionales de España: La Transición (1975-78) El tratamiento informativo y la percepción exterior de la política española en la prensa internacional" (CSO2009-09655), financiado por el Ministerio de Ciencia e Innovación de España.
\end{abstract}




\section{Referencia normalizada}

RODRÍGUEZ-MARTÍNEZ, RUTH; TULLOCH, Christopher; y GUILLAMET LLOVERAS, Jaume (2015): "La muerte de Franco y la Transición española a través de la prensa internacional: la visión periodística del Reino Unido, Francia, Italia y Estados Unidos". Estudios sobre el Mensaje Periodístico. Vol. 21, Núm. 1 (enero-junio), págs.: 193-205. Madrid, Servicio de Publicaciones de la Universidad Complutense.

Sumario: 1. Introducción. 2. Objetivos y metodología. 3. Resultados. 4. Conclusiones. 5. Referencias bibliográficas

\section{Introducción}

La muerte del Generalísimo Franco en noviembre de 1975 y la incógnita sobre lo que sucedería tras su ausencia suscitó un gran interés entre la prensa internacional. La situación de España en este momento era extremadamente crítica, ya que el régimen dictatorial creado al final de la Guerra Civil parecía no ofrecer suficientes garantías para su conversión en un sistema democrático. En primer lugar, el Príncipe Juan Carlos era nombrado por Franco y representaba una monarquía de nueva instauración que debía continuar su régimen, por lo que no gozaba de apoyos suficientes en el interior ni en el exterior. En segundo lugar, los sectores más conservadores del ejército, las fuerzas de seguridad y la administración se oponían a cualquier cambio democrático. Prueba de ello es la ejecución de cinco penas de muerte por terrorismo el 25 de septiembre de 1975, desoyendo el clamor internacional por el indulto. El tercer aspecto a tener en cuenta es la ausencia de una oposición moderada organizada, lo que no favorecía el pluralismo político. Además, la Iglesia Católica se alejaba progresivamente del oficialismo a favor de un clima de moderación general, lo que la enfrentaba con los sectores más intransigentes. Por último, el aspecto económico también resultó determinante ya que, a diferencia de Portugal, España era un país en vías de desarrollo con una economía fuertemente ligada a la inversión extranjera y a los ingresos proporcionados por el turismo.

La inestabilidad manifiesta se tradujo en un gran despliegue de corresponsales y enviados a nuestro país como no sucedía desde el fin de la guerra civil (Knightley, 2000; Southworth, 1977; Preston, 2008). Del mismo modo que numerosas crónicas escritas durante la guerra han quedado como fuente histórica de algunos de sus pasajes más discutidos, diversos autores inciden en el papel similar que ejerció la prensa internacional durante la Transición (Graham, 1984, Hooper, 1995; Aguilar, 2007, Chislett, 2011). El papel desempeñado por la prensa extranjera en el proceso de transición español se enmarca con estudios previamente publicados sobre la función fundamental que desempeñan los medios de comunicación en procesos de consolidación democráticos. Un gran número de investigaciones apoyan esta idea (Schmitter, 1986; Pridham, 1991) independientemente del ámbito geográfico, ya que existen estudios comparativos de procesos democráticos de Latinoamérica, Sudáfrica, Rusia y Europa del Este (Filgueira and Nohlen 1994; Anderson, 1999; Jones, 2001; Gross, 2004; Voltmer, 2006).

En el caso de la Transición española a la democracia, gran parte de los protagonistas del proceso (Areilza, 1977; Fraga, 1987) e historiadores (Powell, 1991, 2001; Preston, 2003; Tusell, 2005; Fuentes, 2011) no dudan en considerar la prensa como un 
elemento fundamental que contribuyó a la evolución y resultado de este proceso (Powell, 1994). Pero si bien la Transición ha sido analizada por numerosos estudiosos durante las tres últimas décadas (Carr and Fusi 1993; Maravall, 1982; Preston 1986; Tuñon de Lara, 1991; Tusell 1996, 1999; Tezanos et al 1993; Soto, 1998; Gallego, 2008 ...), diversos autores apuntan a que la cobertura que hicieron los periodistas internacionales sobre este hecho histórico merece mayor atención (Barrera, 1995, 1997; Guillamet, 1996; Fuentes and Fernández Sebastián, 1997; Barrera and Zugasti, 2006; Saiz y Seoane, 2006; Zugasti, 2008; Quirosa Cheyrouza y Muñoz, 2009). La aparición en los últimos años de los testimonios de los corresponsales (Haubrich, 2009; Chislett, 2011), diversos estudios sobre las potencias europeas respecto a la Transición (Martin Garcia and Ortiz, 2010) o el análisis del papel jugado por la prensa de Estados Unidos (Powell, 2011), ha contribuido a reparar esta carencia, pero quedan aún numerosas cuestiones por ser estudiadas. Este artículo se propone analizar el alcance, importancia e influencia de la cobertura realizada por once diarios internacionales de élite desde la muerte de Franco en 1975 hasta diciembre de 1978.

\section{Objetivos y metodología}

El objeto de estudio de esta investigación son todas las noticias -desde textos estrictamente informativos hasta editoriales- publicadas por los diarios internacionales seleccionados que tienen relación con la Transición. De igual modo, se tienen en cuenta las aportaciones informativas propias de cada cabecera con el fin de detectar la singularidad de cada rotativo. De esta manera se pueden observar las posiciones políticas expresadas por los periódicos estudiados respecto a la Transición, así como su evolución en los tres años en los que se enmarca este proceso. Nuestro interés por la cobertura realizada por la prensa extranjera tiene como punto de partida las siguientes ideas:

1. La cobertura periodística de estos diarios puede proporcionar una visión particular de un hecho histórico, que matice e incluso modifique las visiones anteriores dadas por la historiografía sobre el período. La distancia crítica de la prensa extranjera en relación con los hechos y las luchas domésticas proporciona una perspectiva diferente.

2. La prensa internacional muestra un claro interés por España debido a motivos políticos, estratégicos, económicos y sociales. A estos motivos se añade el temor del estallido de un segundo conflicto bélico que comprometa una transición pacífica a un régimen democrático.

3. El fin de la dictadura española se une a otros cambios sociopolíticos de primera magnitud como la caída de la dictadura militar en Grecia, la revolución de Portugal, el ascenso del eurocomunismo, el terrorismo de izquierdas en Italia y Alemania y el terrorismo nacionalista irlandés en Reino Unido. Los diarios de los países estudiados se interesan, por tanto, en el proceso democrático que tiene lugar en España y resto de países mencionados ya que lo que ocurra en estos países puede afectar a la estabilidad y equilibrio de fuerzas de la Guerra Fría. 
Para observar la cobertura realizada por la prensa extranjera se ha hecho una selección del período histórico que comprende los tres años transcurridos entre la coronación del rey Juan Carlos I de Borbón, el 22 de noviembre de 1975, y la aprobación en referéndum de la Constitución democrática, el 6 de diciembre de 1978. Sin discutir ni rechazar otras concepciones temporales que amplían este periodo transitorio hasta la victoria del PSOE en 1982 o, incluso, hasta 1986 con el ingreso de España en la CEE, se ha considerado el período indicado como el más idóneo para la investigación. Esto se debe a que, a partir de la promulgación de la Constitución, la intensidad de la cobertura de los diarios analizados y la presencia de corresponsales y enviados especiales desciende notablemente.

En lo que respecta a la muestra utilizada para analizar esta cobertura, se ha hecho una selección de los principales diarios de reconocido prestigio de las potencias más interesadas en el proceso español y más ligadas a su historia contemporánea, esto es: Francia, Reino Unido, Estados Unidos e Italia. Se han seleccionado los diarios de referencia y de mayor cobertura sobre el proceso español en cada país, de acuerdo con los criterios de calidad en la clasificación de los diarios establecidos por Merrill (1980), por lo que los diarios incluidos en la muestra han sido los once siguientes: Le Figaro y Le Monde en Francia; The Times, The Daily Telegraph, The Guardian y The Financial Times en Reino Unido; The New York Times y The Washington Post en Estados Unidos; Corriere della Sera, La Stampa y La Repubblica en Italia.

De acuerdo con el espacio temporal y la muestra indicada, los tres objetivos en los que se ha centrado la investigación han sido los siguientes:

1. Analizar el tipo y volumen de cobertura llevada a cabo por los diarios estudiados desde la muerte de Franco y a través del proceso de transformación del régimen en un sistema democrático.

2. Registrar y comparar los recursos humanos -ya sean corresponsales permanentes o enviados especiales- empleados por cada uno de los periódicos para informar de lo que sucedía en España en los años estudiados.

3. Estudiar la posición editorial de los diarios para observar la existencia de tendencias generales o miradas propias de cada país o cada periódico.

Los criterios principales para la selección de noticias son su relación con las temáticas más recurrentes en los años estudiados: a) apoyo al proceso democrático; b) el papel desempeñado por Juan Carlos I; c) la resistencia del bunker franquista al cambio; d) las denuncias sobre la represión e) la relación con Estados Unidos y Europa; f) el terrorismo, g) las demandas de autonomía; h) Adolfo Suárez y el proceso de reformas; i) la legalización del Partido Comunista; j) el fantasma de la Guerra Civil; k) los problemas económicos y, en especial, las cuestiones vinculadas a la inversión extranjera y el turismo. Cuestiones de carácter bilateral como las relaciones con Francia a propósito del Sahara o con el Reino Unido sobre Gibraltar, han sido excluidas de la muestra.

Como herramienta metodológica se ha desarrollado una base de datos en la que se han recopilado las noticias publicadas durante los 1080 días que han sido objeto de estudio por los diarios elegidos en los que se detallan los siguientes aspectos: titular, 
subtítulo, lenguaje, autor, fecha, género, día de la semana, sección, página de publicación, extensión, temas abordados y protagonistas de cada uno de los textos. La diversidad de uso de los géneros periodísticos en los países estudiados, especialmente en el terreno de la opinión, ha obligado a adoptar criterios prácticos de selección de los textos de acuerdo con sus respectivas tradiciones informativas.

Como complemento a este análisis ha sido fundamental la realización de entrevistas en profundidad en París, Londres, Madrid y Roma a los corresponsales y enviados especiales que cubrieron la Transición. Asimismo, con el fin de acceder a los ejemplares originales de los diarios estudiados se han visitado los fondos hemerográficos del Institut d'Études Politiques de París, British Library de Londres, London School of Economics, Universidad de Columbia de Nueva York, Luciano Lama de Roma y Archivo General de la Administración de Alcalá de Henares.

\section{Resultados}

El análisis cuantitativo de las noticias publicadas sobre la Transición en los periódicos estudiados muestra que los principales periódicos de Francia, Reino Unido, Estados Unidos e Italia realizaron una cobertura que responde a las siguientes características:

- Continua: basada en el seguimiento permanente de la vida política española a lo largo de más de mil días

- Regular: con un ritmo ininterrumpido y sin vacios temporales

- Intensa: gracias a la asignación importante y generosa de recursos humanos y espacio en los diarios

- Completa: por incluir todo tipo de enfoques, géneros periodísticos y fuentes informativas

- Personalizada: caracterizada por el envío de corresponsales propios que ofrecen crónicas de mayor cercanía y el uso limitado de despachos de agencia.

TABLA 1. Cobertura de la Transición en los diarios estudiados desde noviembre 1975 a diciembre 1978

\begin{tabular}{|c|l|c|c|c|c|}
\hline Pais & \multicolumn{1}{|c|}{ Diario } & Corresponsales & Enviados & Noticias & EdITORIALEs* \\
\hline \multirow{3}{*}{ Francia } & Le Monde & 3 & 2 & 852 & 56 \\
\cline { 2 - 6 } & Le Figaro & 1 & 3 & 332 & 1 \\
\hline \multirow{5}{*}{ UK } & The Times & 2 & 2 & 1.261 & 36 \\
\cline { 2 - 6 } & $\begin{array}{l}\text { The Daily } \\
\text { Telegraph }\end{array}$ & 4 & - & 663 & 25 \\
\cline { 2 - 6 } & The Guardian & 3 & 2 & 816 & 32 \\
\cline { 2 - 6 } & $\begin{array}{l}\text { The Financial } \\
\text { Times }\end{array}$ & 3 & 2 & 867 & 25 \\
\hline \multirow{5}{*}{ Italia } & $\begin{array}{l}\text { Il Corriere della } \\
\text { Sera }\end{array}$ & 1 & - & 764 & 5 \\
\cline { 2 - 6 } & La Stampa & - & 3 & 456 & 4 \\
\cline { 2 - 7 } & La Repubblica & 1 & 3 & 610 & 8 \\
\hline
\end{tabular}




\begin{tabular}{|c|l|c|c|c|c|}
\hline \multirow{2}{*}{ EEUU } & $\begin{array}{l}\text { The New York } \\
\text { Times }\end{array}$ & 2 & 2 & 648 & 14 \\
\cline { 2 - 6 } & $\begin{array}{l}\text { The Washington } \\
\text { Post }\end{array}$ & 2 & - & 388 & 6 \\
\hline TOTAL & 11 & 22 & 19 & 7.657 & 212 \\
\hline
\end{tabular}

*El número de editoriales está incluido en el total de las noticias contabilizadas

De acuerdo con los resultados que pueden observarse en la tabla se destacan, entre otros, los siguientes factores:

i) El diario que destaca de forma notoria en cuanto al número total de noticias publicadas es el británico The Times. Su promedio de publicación (1.261 piezas) es superior a una pieza diaria, hecho destacable dada la extensión de la muestra, que conformada con un total de 1080 días de análisis. Es preciso señalar el prolífico ritmo de publicación del diario británico, ya que supera en más de 400 noticias al segundo periódico más productivo: el francés Le Monde.

ii) Los resultados ponen de manifiesto el interés general que despierta el proceso democrático español en la prensa del Reino Unido. De las cinco cabeceras que superan la media de publicación de noticias, calculada en 696 noticias, tres son de Londres: The Times, The Guardian y The Financial Times.

iii) Un tercer resultado se refiere a la desigualdad cuantitativa entre los diarios de todos los países analizados. Este es el caso de Francia, donde Le Monde publica más del doble de noticias que Le Figaro. Esta diferencia también se aprecia en el caso italiano: el número de noticias de Il Corriera della Sera supera en un $60 \%$ el número de piezas publicado por La Stampa. La cobertura de Estados Unidos guarda similitud con lo que ocurre en Francia y, sobre todo, Italia, ya que el diario de Nueva York también publica un $60 \%$ más de noticias que su homólogo capitalino.

iv) En lo que respecta al despliegue de recursos humanos es preciso destacar que, con sólo dos excepciones -Corriere della Sera y The Washington Post- todos los diarios de la muestra utilizaron más de tres reporteros para informar sobre la Transición. El país que cuenta con menor número de corresponsales y enviados especiales para cubrir la Transición es Italia, ya que de las tres cabeceras estudiadas tan sólo han sido contabilizados dos corresponsales y seis enviados especiales.

v) La suma del número de editoriales publicados durante el periodo estudiado asciende a un total de 212 piezas. Es preciso destacar que, de todos ellos, Le Monde es el diario que más utiliza este recurso para proyectar su opinión sobre lo que sucede en España. La cifra de 56 editoriales publicados en Le Monde es muy superior al del resto de la muestra ya que constituye un $26 \%$ del global de editoriales publicados. Este número de editoriales representa el seis por ciento del total de piezas aparecidas en el rotativo francés, ratio claramente superior a cualquier otro diario de la muestra.

Una vez definidos los resultados cuantitativos de la muestra, se procede a destacar aspectos de carácter cualitativo. Para este tipo de análisis se tiene en cuenta exclusivamente el análisis de los editoriales publicados. Esto se debe a la ascendencia de este género en relación con los otros componentes informativos de un diario para sintetizar la postura del medio ante cualquier suceso relevante. Los editoriales se pueden 
clasificar en tres categorías: a) los editoriales que abordan la muerte de Franco, la coronación de Juan Carlos y procesos como el referéndum y las elecciones legislativas generan un total de 40 editoriales, esto es, casi uno de cada cinco editoriales publicados; b) editoriales que prestan atención a las medidas gubernamentales tomadas por Carlos Arias Navarro y Adolfo Suárez ocupan un total de 84 piezas dedicadas; c) los 88 editoriales restantes se refieren a los aspectos más conflictivos y complejos del proceso. En cuanto a ellos, se puede hacer la siguiente subclasificación: 19 editoriales están dedicados al proceso de legalización del Partido Comunista; 17 textos se refieren a actos violentos o terroristas (siete de ellos cubren el asesinato en enero de 1977 de los abogados de Atocha), dieciséis piezas versan sobre actos vinculados con represión policial; 16 editoriales abordan las demandas de autonomía de Cataluña y el País Vasco; ocho piezas tratan la cobertura de movimientos obreros o huelgas; y, por último, cuatro editoriales están dedicados a la concesión de la amnistía.

En lo que se refiere a la temática de estos editoriales, hay diversos temas que son tratados de forma recurrente y conforman el hilo conductor del proceso democrático. Se pueden destacar, entre otros, los siguientes:

1. El fantasma de la guerra civil: En raras ocasiones la guerra civil aparece como tema principal, pero su fantasma sobrevuela de forma intermitente en la cobertura de la prensa internacional, desde antes de la muerte de Franco y durante todo el proceso democrático. Lo evocan especialmente los diarios británicos en ocasión del recrudecimiento de la violencia y las cinco penas de muerte por terrorismo ejecutadas en septiembre de 1975 y es un riesgo que también ve Le Monde en Francia. Diarios de otros países como Italia y Estados Unidos también muestran su temor ante la violencia política del terrorismo y la propia policía, así como el malestar en el ejército que tiene a España en vilo antes y después de las elecciones legislativas en junio de 1977.

2. Apoyo a la democratización: Hay un firme y general apoyo a la instauración de un régimen democrático que permita la plena integración de España en las alianzas políticas y militares de Europa Occidental. No hay unanimidad en Estados Unidos en cuanto a la velocidad del cambio. De acuerdo con las reticencias de la administración Ford-Kissinger, The Washington Post está a favor de un proceso lento, a diferencia de The New York Times que, como los diarios europeos, aboga por cambios rápidos. La fuerte represión policial de inicios de 1976, sobretodo en Madrid, Barcelona y Vitoria a inicios de 1976, acentúa el rechazo de los intentos de perpetuar el franquismo.

3. El papel del rey Juan Carlos I: La figura del monarca funciona de termómetro para evaluar la viabilidad del proceso democrático. Salvo The Daily Telegraph y The Washington Post, que muestran su confianza desde el primer día, hay una evolución en los demás diarios desde las reservas y la decepción inicial a la aceptación absoluta del papel jugado por Juan Carlos de Borbón. La idea de que el rey tiene el trono pero no el poder, la confirmación del primer ministro franquista Carlos Arias Navarro y la no concesión de una amnistía o apertura política perjudican la percepción sobre su capacidad y voluntad democrática. Se le presenta como un monarca desconocido por la población, despreciado por el búnker franquista, rechazado por la oposición y sólo apoyado por el Ejército, del que es comandante en jefe. Esta percepción cambia progresivamente, especialmente con motivo de su viaje a Estados Unidos. Éste es el punto 
de inflexión, ya que el rey pasa a ser visto como el garante de las reformas y de la democracia en España y todas las críticas se centran en Arias Navarro.

4. Bunker franquista: La resistencia de la extrema derecha franquista, conocida como el búnker, y su capacidad para poner obstáculos al cambio democrático es denunciada desde los primeras semanas del gobierno continuista de Arias Navarro. La prensa italiana califica a la extrema derecha española como el máximo peligro para la reforma y denuncia sus vínculos con los grupos fascistas violentos de su país. La prensa británica señala su atrincheramiento en la Administración pública, el Movimiento Nacional (partido único franquista) y las Fuerzas Armadas. La progresiva oposición de los altos jefes militares a la reforma convierte al ejército y a la policía en el principal reducto del búnker, incluso después del referéndum de la reforma política y de las primeras elecciones democráticas.

5. Apoyo total a la reforma de Suárez: El tándem que a partir de julio de 1976 forma el rey Juan Carlos I con el joven primer ministro Adolfo Suárez, que ejecuta rápidamente un programa de reformas, obtiene el apoyo inmediato de la prensa internacional. Para la prensa extranjera de izquierdas, Suárez es el hombre designado para desmontar desde dentro el sistema franquista, mientras que para la conservadora ofrece la oportunidad de crear un centro político. Hay coincidencia en señalar que tanto el Rey como Suárez eran ajenos a la experiencia de la guerra civil, como lo son a la situación de violencia extremista creciente. El triunfo de las dos grandes opciones moderadas y la desautorización simultánea del franquismo y del comunismo son vistos por los diarios como una prueba inesperada de la madurez política española y garantía de estabilidad. The Financial Times no deja de reprochar a Suárez el aplazamiento constante de reformas económicas, hasta el pacto social de la Moncloa de octubre. El francés Le Figaro valora el centrismo de Suárez como la mejor manera de que España exorcice los demonios del extremismo y la discordia. Sólo la prensa italiana desconfía del tándem reformista Juan Carlos-Suárez, especialmente La Repubblica, hasta la legalización del PCE.

6. Legalización del PCE: Desde el primer momento de la monarquía reinstaurada, la prensa internacional exige, sin excepciones, la legalización del Partido Comunista de España, como condición sine qua non para el reconocimiento de la democracia española. La exigencia es mayor en la prensa de Italia y Francia, con presencia comunista importante en el parlamento, pero no es menos firme en la de Reino Unido y Estados Unidos, donde ni siquiera la hay. En la prensa italiana, Santiago Carrillo es la voz más autorizada de la oposición y La Stampa llega a calificar al PCE como el partido eurocomunista más avanzado y antisoviético. La prensa angloamericana constata las diferencias entre el duro comunismo portugués y el pacífico comunismo español. La legalización del PCE en abril de 1977, en víspera de la convocatoria de elecciones, es valorada como el paso definitivo hacia la democracia.

7. Las autonomías de Cataluña y País Vasco: La comprensión general de las demandas de autonomía de Cataluña y el País Vasco, fundadas en razones históricas, culturales y lingüísticas, choca con las dificultades que plantean, distintas en cada caso. Hay mayores reservas en la prensa norteamericana y un más fuerte apoyo en la italiana, que llega a plantear una estructura federal para la democracia española. El 
conflicto con la monarquía que plantea la demanda catalana de restablecimiento del Estatuto de Autonomía republicano de 1932 es resuelto por sorpresa con el pacto de Suárez con el presidente de la Generalitat en el exilio, Josep Tarradellas, poco después de las elecciones. El británico The Times describe el retorno de éste a Barcelona como un momento significativo del siglo XX en Europa. En cambio, la falta de entendimiento del gobierno con el Partido Nacionalista Vasco para la elaboración de la Constitución, la división de los partidos y la espiral creciente de violencia terrorista y policial, imposibilita una solución. La prensa británica, en especial, advierte del peligro que ello suponga un cierre en falso de la Transición, que se confirma con el bajo voto favorable que recibe la nueva Carta Magna entre los vascos.

8. Terrorismo heredado y agravado: El terrorismo vasco de ETA y de extrema izquierda de FRAP es valorado inicialmente como una herencia de la dictadura y su falta de libertades políticas, así como nuevos grupos como GRAPO, sospechoso de infiltración policial, y los fascistas de extrema derecha vinculados al búnker. La prensa británica es comprensiva con el terrorismo vasco e incluso la italiana, pese a su experiencia paralela con las Brigadas Rojas, que en mayo de 1978 asesinarán al primer ministro democristiano Aldo Moro. También la francesa, dada la cantidad de refugiados activos que viven en Francia protegidos por los acuerdos de Ginebra. A medida que avanza la reforma, ETA se confirma como la principal amenaza para el cambio democrático, pero la prensa británica e italiana no deja de señalar el amplio apoyo popular que recibe. A ello se añade la denuncia de la mala gestión política del problema vasco por parte del gobierno, con una policía que actúa de manera brutal e indiscriminada contra la población.

9. OTAN y CEE. El interés de las grandes potencias por la democratización española se relaciona con la posible integración en las alianzas políticas y militares de la Europa Occidental, aún en el contexto de la guerra fría. La perspectiva europea respecto al ingreso de España en la OTAN es importante, como analiza Le Monde, por la inestabilidad resultante de la revolución en Portugal y la incertidumbre en Italia, con un partido comunista muy cerca de la entrada en el gobierno. España es un importante bastión para Estados Unidos, de forma que los dos diarios estadounidenses analizados apoyan los acuerdos militares con España a principios de 1976, frente a las críticas de algunos diarios europeos por su precipitación, que comparte The New York Times. La prensa expone la posición internacional sobre el ingreso de España en la CEE de forma no siempre coincidente con sus gobiernos. El apoyo general al ingreso como forma de proteger la Transición española, expresado también por los diarios de Francia e Italia, contrasta con los problemas de competencia que este ingreso plantearía para la agricultura de ambos países. La posición del gobierno francés es muy tajante, frente al interés italiano de tener a Grecia, Portugal y España como aliados para frenar la muy subvencionada agricultura del norte. La posición es más favorable en Gran Bretaña, donde The Times enarbola con más intensidad la bandera europeísta, coincidiendo con el interés británico por una comunidad más grandes y flexible. Cuando en julio de 1977, España presenta formalmente la solicitud de ingreso, The Financial Times señala los problemas que la adhesión plantea internamente a la economía española y como diario económico se extiende en un análisis sistemático de las mismas. 


\section{Conclusiones}

La presente investigación se propone observar la Transición española desde la perspectiva de la prensa internacional con el fin de comprender la manera en que los corresponsales y enviados especiales llegados desde el extranjero narraron este proceso político. Este enfoque toma como referencia el trabajo realizado previamente por diversos autores (Graham, 1984, Hooper, 1995; Aguilar, 2007, Chislett, 2011) y ofrece resultados concretos sobre la cobertura realizada por los principales diarios de Reino Unido, Francia, Italia y Estados Unidos.

Ante la incertidumbre que supuso la muerte de Franco y el temor del estallido de un nuevo conflicto bélico, los diarios internacionales no dudaron en enviar un gran número de periodistas para cubrir todo lo que ocurría en el territorio español. Esto explica el alto interés que los periódicos estudiados prestaron a España, lo que se tradujo en una cobertura continua, regular, intensa, completa y personalizada. El total de noticias publicadas en los tres años analizados alcanza la cifra de 7.657, por lo que las noticias sobre España eran más que habituales en las portadas y secciones internacionales de los rotativos analizados.

Los resultados cuantitativos de la investigación permiten apreciar el gran interés que suscita el proceso de la Transición en países como el Reino Unido. El número de noticias que aparecen en las páginas de The Times, The Guardian y The Financial Times supera la media de publicación. De hecho, el periódico que hace la cobertura más extensa sobre la Transición es The Times, cuya frecuencia de publicación supera una pieza diaria en el periodo analizado. El papel que juega el Rey durante todo el proceso de cambio democrático no deja indiferente a los periódicos británicos, habituados a tratar la institución monárquica como hecho informativo, a diferencia del resto de países estudiados. Otras cuestiones que pueden explicar esta extensa cobertura son: por un lado el contencioso con Gibraltar, que vive momentos de tensión durante el proceso de la Transición; y, por otro lado, el temor ante una nueva Guerra Civil, hecho que ha acaparado históricamente el interés de los diarios británicos a lo largo del régimen franquista.

Otro de los aspectos que se han tenido en cuenta en la presente investigación es destacar el número total de editoriales publicados por los diarios estudiados, que asciende a 212. De todas las cabeceras de la muestra, el periódico que más editoriales publica es el francés Le Monde. Sus 56 editoriales, cuya frecuencia de publicación es de un editorial cada veinte días, hacen que este diario sume el $26 \%$ del global de editoriales registrados. El interés ideológico del liberal Le Monde en apoyar el proceso democrático para poner fin a una dictadura conservadora en el país vecino puede explicar este dato. Asimismo, la importancia de la opinión frente a los hechos en los diarios franceses también puede ayudar a comprender la intensa toma de posición mostrada por este periódico. Esto contrasta con la poca presencia de editoriales registrada en $L e$ Figaro, más próximo a posiciones conservadoras y, como consecuencia, menos críticos con el régimen postfranquista. En cambio, en lo que respecta a la prensa británica la diversidad ideológica de los diarios seleccionados, que abarca desde The Guardian hasta The Daily Telegraph, no es un factor que condicione el volumen de publicación de editoriales. 
Por otro lado, el interés que suscita España en los países estudiados queda patente en el importante despliegue de recursos humanos en forma de corresponsales y enviados especiales que llegan para cubrir la Transición. El estudio revela que los diarios analizados, a excepción del Corriere della Sera y The Washington Post, contaban con una media de cuatro periodistas para cubrir lo que ocurría en España durante los tres años estudiados.

Desde el punto de vista cualitativo, los temas sobre los que los diarios estudiados se posicionan con mayor frecuencia son, entre otros, los siguientes: el apoyo al proceso de democratización que ponga fin a la dictadura; el papel desempeñado por el Rey y Adolfo Suárez para garantizar la paz durante el proceso de cambio político; la legalización del PCE como condición indispensable para garantizar el éxito de la Transición; las demandas autonómicas de Cataluña y el País Vasco, en el que se destaca la dificultad de encontrar una solución al terrorismo vasco frente a la actitud más moderada de Cataluña.

Los resultados derivados de esta investigación ayudan a clarificar y comprender mejor la manera en que la prensa internacional cubrió la Transición española. Si bien los resultados derivan de una muestra de cerca de más de siete mil ítems de análisis, los autores son conscientes de la restricción de la muestra a un reducido número de países o a una determinada tipología de diarios.

Con el fin de comparar y comprender las diferencias y similitudes existentes entre la labor realizada por los periodistas extranjeros y españoles en el mismo periodo y espacio, el grupo de investigación que ha llevado a cabo este análisis está realizando actualmente una investigación sobre la cobertura que los diarios españoles hicieron de la Transición.

\section{Referencias bibliográficas}

AGUILAR, Miguel Ángel (2007): "Harry Debelius, periodista" en El País, 20 de febrero, sección Necrológica.

ANDERSON, Lisa (1999): Transitions to Democracy. New York, Columbia University Press.

AREILZA, José María (1977): Diario de un ministro de la monarquía. Barcelona, Planeta.

BARRERA, Carlos (1995): Sin mordaza. Veinte años de prensa en democracia. Madrid, Temas de hoy.

BARRERA, Carlos (1997): "Poder político, empresa periodística y profesionales de los medios en la transición española”. Comunicación y Sociedad, Volumen X, número 2. Navarra, Facultad de Comunicación, pp. 7-46.

BARRERA, Carlos y ZUGASTI, Ricardo (2006): "The role of the press in times of transition: The building of the Spanish democracy (1975-78)" en Voltmer, Katrin (Ed.): Mass media and political communication in new democracies. New York, Routledge, pp. 23-41.

CARR, Raymond y FUSI, Juan Pablo (1993): Spain: Dictatorship to democracy. Barcelona, Planeta. 
CHISLETT, William (2011): The Foreign Press During Spain's Transition to Democracy, 1974-78. A Personal Account. Madrid, Fundación Transición Española.

FILGUEIRA, Carlos y NOHLEN, Dieter (1994): Prensa y transición democrática. Experiencias recientes en Europa y América Latina. Madrid, Iberoamericana.

FRAGA IRIBARNE, Manuel (1987): En busca del tiempo servido. Barcelona, Planeta.

FUENTES, Juan Francisco y FERNÁNDEZ SEBASTIÁN, Javier (1997): Historia del periodismo español. Madrid, Síntesis.

FUENTES, Juan Francisco (2011): Adolfo Suárez, biografía política. Barcelona, Planeta.

GALLEGO, Ferrán (2008): El mito de la transición. La crisis del franquismo y los orígenes de la democracia (1973-1977). Barcelona, Crítica.

GRAHAM, Robert (1984): Spain. The Change of a Nation. London, Michael Joseph.

GROSS, Peter (2004): "Between Reality and Dream: Eastern European Media Transition, Transformation, Consolidation and Integration" en East European Politics \& Societies. Volumen 18, número 1, pp.110-131.

GUILLAMET, Jaume (1996): Premsa, franquisme i autonomia. Barcelona, Flor del Vent.

HAUBRICH, Walter (2009): "La Transición española en la prensa alemana. El caso de Frankfurter Allgemeine Zeitung". En QUIROSA CHEYROUZA Y MUÑOZ, Rafael: Prensa y Democracia. Los medios de comunicación en la Transición. Madrid, Biblioteca Nueva, pp. 297-304.

HOOPER, John (1995): The New Spaniards. London, Penguin.

JONES, Adam (2001): The Press in Transition A Comparative Study of Nicaragua, South Africa, Jordan, and Russia. Hamburg, Deutsches Übersee-Institut.

KNIGHTLEY, Philip (2000): The First Casualty. The War Correspondent as hero and myth-maker, from the Crimea to Kosovo. London, Prion.

MARAVALL, José (1982): The Transition to Democracy in Spain. London, Croom Helm.

MARTÍN GARCÍA, Oscar José y ORTIZ, Manuel (2010): Claves Internacionales en la Transición Española. Madrid, Catarata.

MERRILL, John \& FISHER, Harold (1980): The world's great dailies. Profiles of fifty newspapers. New York, Hastings House.

POWELL, Charles (1991): El piloto del cambio: el rey, la monarquía y la transición a la democracia, Barcelona, Editorial Planeta.

POWELL, Charles (1994): "La dimensión exterior de la transición política española" en Revista del Centro de Estudios Constitucionales. Número 18, pp. 79-116.

POWELL, Charles (2001): España en democracia, 1975-2000. Barcelona, Plaza y Janés. 
POWELL, Charles (2011): El amigo americano. España y Estados Unidos: de la dictadura a la democracia. Madrid, Galaxia Gutenberg.

PRESTON, Paul (1986): The Triumph of Democracy in Spain 1969-1982. London, Methuen.

PRESTON, Paul (2003): Juan Carlos. El rey de un pueblo. Barcelona, Plaza y Janés.

PRESTON, Paul (2008): We Saw Spain Die. Londres, Constable and Robinson.

PRIDHAM, Geoffrey (1991): Encouraging democracy. The International Context of Regimen Transition in Southern Europe. Leicester, Leicester University Press.

QUIROSA-CHEYROUZA Y MUÑOZ, Rafael (2009): Prensa y Democracia. Los medios de comunicación en la Transición. Madrid, Biblioteca Nueva.

SAIZ, María Dolores y SEOANE, María Cruz (2006): Cuatro siglos de periodismo en España. Madrid, Alianza.

SCHMITTER, Philippe (1986): “An Introduction to Southern European Transitions from Authoritarian Rule: Italy, Greece, Portugal, Spain” en O'Donnell, Guillermo, Schmitter, Philippe y Whitehead, Laurence (Eds.): Transitions from authoritarian rule. Baltimore, MD, Johns Hopkins University.

SOTO, Álvaro (1998): La transición a la democracia. España 1975-1982. Madrid, Alianza.

SOUTHWORTH, Herbert Rutledge (1977): Guernica! Guernica! A Study of Journalism, Propaganda and History. Berkeley, University of California Press.

TEZANOS, José Félix; COTARELO, Ramón; y DE BLAS, Andrés (eds., 1993): La transición democrática española. Madrid, Editorial Sistema.

TUÑÓN DE LARA, Manuel (1991): Historia de España. Volumen 10/2 Transición y democracia: 1973-1985. Barcelona, Ed. Labor.

TUSELL, Javier (1996): Historia de la Transición (1975-1986). Madrid, Alianza.

TUSELL, Javier (1999): La transición española a la democracia. Madrid, Historia 16.

TUSELL, Javier (2005): Dictadura franquista y democracia, 1939-2044, Historia de España, XIV, Barcelona, Crítica.

VOLTMER, Katrin (Ed., 2006): Mass media and political communication in new democracies. New York, Routledge.

ZUGASTI, Ricardo (2008): "El papel de la prensa en la construcción de la democracia española: de la muerte de Franco a la Constitución de 1978" en Confines de Relaciones Internacionales y Ciencia Política. Volumen 4, número 7, pp. 53-68. 